\title{
Bolsas de ensino e pesquisa para a Educação Física no Brasil concedidas pelo CNPq: análise temporal de 2005 a 2014
}

\author{
Scholarships of teaching and research for Physical Education in Brazil \\ granted by CNPq: a temporal analysis of 2005 to 2014
}

\section{Leony Morgana Galliano; : Leandro Quadro Corrêa1,2; Antônio Evanhoé Pereira de Souza Sobrinho 1,3; Daiana Lopes de Rosa; Francisco Marlon da Silva Gomes"; Marceli Pureza de Melo5, Marlos R Domingues', Pedro C Hallal'}

\section{Resumo}

Este estudo objetivou identificar as verbas por tipo e modalidade de bolsas de ensino e pesquisa concedidas pelo CNPq entre 2005 e 2014 nas grandes áreas de conhecimento, especialmente a de Ciências da Saúde com foco na subárea da Educação Física. As buscas ocorreram no mês de março de 2015 e realizadas no painel de investimentos do $\mathrm{CNPq}$ (http://www.cnpq.br/ painel-de-investimentos) para informações sobre bolsas e no site da CAPES para identificar os cursos de pós-graduação. Foram investidos $\mathrm{R} \$ 813.435 .000,00$ nas diferentes modalidades de bolsa, principalmente produtividade em pesquisa (30,0\%). A área de Ciências da Saúde foi a quinta em termos de recebimento de verbas (11,0\%). Entre as subáreas das Ciências da Saúde, a Medicina foi a que mais recebeu bolsas de ensino e pesquisa $(38,0 \%)$ e a Educação Física ficou em sétimo lugar (4,0\%). As subáreas que mais cresceram em investimentos no período analisado foram a Fisioterapia/Terapia Ocupacional (775,0\%) e a Educação Física (738,0\%), sendo esta última a sétima das subáreas em recebimento de bolsas de produtividade. Entretanto, foi a quarta em termos de crescimento de investimentos. Do total de bolsas de ensino e pesquisa, $64,3 \%$ foram destinadas a pesquisadores da região Sudeste, $15,4 \%$ na região Sul e $14,6 \%$ no Nordeste. Em contrapartida, apenas 4,1\% para a região Centro-oeste e $1,6 \%$ a pesquisadores da região Norte.

\section{Palavras-chave}

Investimentos; Pesquisa; Educação; Brasil

\begin{abstract}
The aim of the present study was to evaluate funding of the Brazilian Research Council (CNPq) via scholarships between 2005 and 2014 in Health Sciences and particularly in the sub-area of Physical Education. The search for scholarships was carried out in March, 2015 in the investments panel of the CNPq website (http://www. cnpq.br/painel-de-investimentos), as well as in the CAPES website to identify programs. Total funding was BRL 813,435,000.00; the main type of scholarship was the "research productivity" (30.0\%). Health Sciences field placed fifth according to the total amount (11.0\%). Within this field, Medicine received the biggest share of scholarships and funding (38.0\%) while Physical Education placed seventh (4.0\%). The sub-areas with the highest increases in funding were Physiotherapy/Occupational Therapy (775.0\%) and Physical Education (738.0\%). Physical Education ranked seventh in the receipt of productivity scholarships, but was the fourth in terms of growth in the number of scholarships. Of all scholarships, $64.3 \%$ were given to researchers in the Southeast region, $15.4 \%$ to South region and $14.6 \%$ to Northeast. On the other hand, only $4.1 \%$ were awarded to researchers from Center-west region and $1.6 \%$ to North region.
\end{abstract}

\section{Keywords}

Investment; Research; Education; Brazil

\section{Introdução}

O Conselho Nacional de Desenvolvimento Científico

1 Programa de Pós-Graduação em Educação Física - Universidade Federal de Pelotas ESEF/UFPel, Pelotas, RS

2 Universidade Federal do Rio Grande - FURG, Rio Grande, RS

3 Universidade da Região da Campanha, Bagé, RS

4 Faculdade Atual - Macapá, AP

5 Graduada em Educação Física - Faculdade de Macapá - Macapá, AP e Tecnológico ( $\mathrm{CNPq})$ organiza as grandes áreas de conhecimento em nove categorias, de acordo com a semelhança entre os objetos e métodos utilizados ${ }^{1}$. As grandes áreas de conhecimento são: Ciências Agrárias; Biológicas; Ciências da Saúde; Exatas e da Terra; Humanas; Engenharias e Computação; Línguas, Letras e Artes; Sociais Aplicadas e a classificação de "outras'. Cada uma destas recebe investimentos 
para seu desenvolvimento e esses valores são subdivididos entre suas respectivas subáreas. Dentro de cada grande área existem subáreas, fragmentadas conforme características específicas.

A grande área de Ciências da Saúde constitui-se de nove subáreas do conhecimento (Medicina, Saúde Coletiva, Farmácia, Odontologia, Enfermagem, Nutrição, Fisioterapia e Terapia Ocupacional, Fonoaudiologia e Educação Física) e tem a pesquisa como uma de suas atividades para expansão e fonte de desenvolvimento. A atividade de pesquisa constitui-se em um meio através do qual o corpo de conhecimentos, necessariamente denso e profundo, é lapidado, aperfeiçoado e renovado, realimentando, portanto, o sistema de ensino superior ${ }^{2}$.

$\mathrm{Na}$ Educação Física, a prática da pesquisa no período que decorre entre a inserção de pessoas na universidade e os programas de pós-graduação é condição fundamental no que diz respeito à estratégia de formação, tanto da figura do professor quanto do pesquisador ${ }^{3}$. Apesar disto, a pesquisa nesta área e a sua incorporação no cotidiano é prática contemporânea no Brasil ${ }^{3}$.

O Ministério da Educação, através da Coordenação de Aperfeiçoamento de Pessoal de Nível Superior (CAPES), reconheceu 51 novos cursos de Educação Física (mestrado, mestrado profissional e doutorado) no ano de $2015^{4}$. Além do reconhecimento dos cursos, a CAPES é um dos principais órgãos financiadores de pesquisa, juntamente com o CNPq, o qual publica anualmente editais abertos, sem restrição de áreas do conhecimento no Brasil ${ }^{5}$.

A partir destes editais, são oferecidas bolsas de Iniciação Científica Júnior para os estudantes do ensino médio e bolsas de Iniciação Científica para os alunos de graduação. Além disso, o CNPq oferece bolsas de mestrado, doutorado e doutorado sanduíche no país. Dentro das bolsas ofertadas no exterior estão as bolsas de graduação sanduíche, doutorado pleno, doutorado sanduíche, pós-doutorado, estágio sênior, treinamento no exterior e Desenvolvimento Tecnológico Inovação Júnior e Sênior em Técnicos em Iniciação Científica. Nas bolsas de auxílio à pesquisa, há o investimento em Projeto Individual de Pesquisa, auxílio para Promoção de Eventos científicos, tecnológico e/ou de inovação, participação em eventos científicos, editoração e pesquisador visitante. Estes investimentos têm como finalidade aperfeiçoar a formação no ensino e pesquisa de alunos de ensino médio, graduação e pós-graduação.

Sabendo que os estudos sobre a destinação de financiamentos de pesquisa podem contribuir em diversos fatores, como a identificação de iniquidades regionais na destinação de verbas, o que permite auxiliar na compreensão do campo de pesquisa e embasar estratégias de evolução na produção do conhecimento e destinação de recursos ${ }^{5}$, o presente estudo teve como objetivo identificar as verbas por tipo e modalidade de bolsas de ensino e pesquisa concedidas pelo CNPq entre 2005 e 2014 nas grandes áreas de conhecimento, especialmente a de Ciências da Saúde com foco na subárea da Educação Física. Não foram realizadas análises de financiamento da CAPES por não se ter acesso a estas informações.

\section{Métodos}

A coleta de dados foi conduzida durante o mês de março de 2015. As informações foram extraídas do Painel de Investimentos do CNPq (http://www.cnpq.br/painelde-investimentos). Este website apresenta dados relacionados aos investimentos por modalidade e tipo de bolsas, além de informações estratificadas pelas grandes áreas do conhecimento (Ciências Exatas e da Terra, Ciências Agrárias, Ciências Biológicas, Ciências da Saúde, Ciências Humanas, Engenharias e Computação, Línguas 
Letras e Artes, Sociais Aplicadas e outras), subáreas, regiões do país e instituições de ensino. Desta forma, obtiveram-se informações sobre investimentos totais de cada grande área e das subáreas das Ciências da Saúde. Assim, foi desenvolvido um estudo descritivo com base em dados secundários sobre a concessão de bolsas e financiamento do CNPq para todas as grandes áreas, entre os anos de 2005 a 2014, destacando a subárea de Educação Física.

Foi realizada análise de distribuição de frequências para verificar os financiamentos em pesquisa e investimentos na Educação Física dentro da área das Ciências da Saúde e para as grandes áreas do conhecimento. Além disso, verificaram-se os investimentos em valores brutos de bolsas das Ciências da Saúde estratificados de acordo com: (a) tipo de bolsa: no país, exterior ou auxílio à pesquisa; (b) modalidade: iniciação científica, mestrado, doutorado, produtividade em pesquisa ou outras; (c) região do país: Norte, Nordeste, Centro-oeste, Sudeste e Sul; (d) subáreas: Medicina, Saúde Coletiva, Farmácia, Odontologia, Enfermagem, Educação Física, Nutrição, Fisioterapia/Terapia Ocupacional, Fonoaudiologia.

Para que fosse feito um panorama relacionando aos investimentos com o número de cursos de pós-graduação (mestrado e doutorado) na área das Ciências da Saúde no país, foram coletadas informações no site da CAPES (http://www.capes. gov.br/cursos-recomendados) e, através de lista obtida neste website, foi verificado o $n^{\circ}$ de cursos e ano de homologação.

São apresentados dados dos financiamentos em valores brutos (reais), bem como através de frequências absolutas e relativas para as variáveis estudadas. Para as análises, foram utilizados os valores apresentados referentes às bolsas no exterior, no país e auxílio a pesquisa para cada grande área e para as subáreas das Ciências da Saúde. Para os cálculos de percentual de crescimento nos últimos 10 anos, utilizou-se o ano de 2005 como linha de base. Os dados foram tabulados e analisados no programa Excel 2013, e as demais análises foram conduzidas no pacote estatístico Stata 10.0.

\section{Resultados}

Entre os anos de 2005 a 2014, a área de "Engenharias e Computação" recebeu, em média, 22,1\% do valor investido em bolsas de ensino e pesquisa, seguida pelas "Ciências Biológicas" (17,9\%) e "Ciências Exatas e da Terra" (16,3\%). A grande área das "Ciências da Saúde" aparece na quinta colocação (11,2\%) (Tabela 1).

Neste período, em média, foram investidos $\mathrm{R} \$ 1.572 .074 .000,00$ por ano nos diferentes tipos de bolsa, sendo o valor total destinado prioritariamente para bolsas no país (51,7\%). Quanto às modalidades de bolsa, em média foram investidos $\mathrm{R} \$ 813.435 .000,00$ por ano, sendo direcionados principalmente para produtividade em pesquisa (30,0\%; Tabela 1). Destaca-se que, de modo geral, houve um aumento no valor destinado à todas modalidades durante o período estudado (dados não apresentados). Nota-se que a partir do ano de 2012, houve diminuição no percentual de bolsas no país e crescimento nas bolsas no exterior (Figura 1). A maior proporção de verba destinada para bolsas no país ocorreu no ano de 2011 (74,4\%). Já para as bolsas no exterior, o maior percentual foi observado em 2014 (24,0\%).

As bolsas de produtividade em pesquisa cresceram 75,0\% entre 2005 e 2014, sendo o curso de Medicina o que mais recebeu recursos para esta modalidade de bolsa (36,0\%), seguido pela Saúde Coletiva (15,0\%). Em contrapartida, ocorreu um crescimento no investimento neste tipo de bolsa naquelas subáreas que recebiam menor proporção de investimentos. A Fisioterapia/Terapia Ocupacional e a Fonoaudiologia foram as subáreas das Ciências da Saúde que receberam menores 
TABELA 1 - Valores anuais médios e proporção de financiamentos por modalidade e tipo de bolsas na área de Ciências da Saúde entre os anos de 2005-2014.

\begin{tabular}{lcc}
\hline Variáveis & Valor em R\$ & $\%$ \\
\hline Tipos de bolsa & & \\
\hline No país & 813.435 .000 & 51,7 \\
\hline No exterior & 148.807 .000 & 9,5 \\
\hline Auxílio & 609.833 .000 & 38,8 \\
\hline Total por tipo de bolsa & 1.572 .074 .000 & 100,0 \\
\hline Modalidades de bolsa & & 30,0 \\
\hline Produtividade em Pesquisa & 244.242 .000 & 18,6 \\
\hline Doutorado & 150.951 .000 & 13,1 \\
\hline Mestrado & 106.419 .000 & 17,7 \\
\hline Iniciação Científica & 144.251 .000 & 20,6 \\
\hline Outros* & 167.572 .000 & 100,0 \\
\hline Total por modalidade & 813.435 .000 & 16,3 \\
\hline Grandes áreas do conhecimento & & 13,5 \\
\hline Ciências Exatas e da Terra & 2.289 .231 & 17,9 \\
\hline Ciências Agrárias & 1.890 .092 & 11,2 \\
\hline Ciências Biológicas & 2.502 .882 & 9,5 \\
\hline Ciências da Saúde & 1.572 .054 & 22,1 \\
\hline Ciências Humanas & 1.328 .842 & 100 \\
\hline Engenharias e Computação & 3.097 .457 & 352.682 \\
\hline Línguas, Letras e Artes & 983.128 & 14.016 .368 \\
\hline Outros & & \\
\hline Total por grande área do conhecimento & \\
\hline
\end{tabular}

*Outros: bolsas de graduação sanduíche, doutorado pleno, doutorado sanduíche, pós-doutorado, estágio sênior, treinamento no exterior e Desenvolvimento Tecnológico Inovação Júnior e Sênior em Técnico em Iniciação Científica.

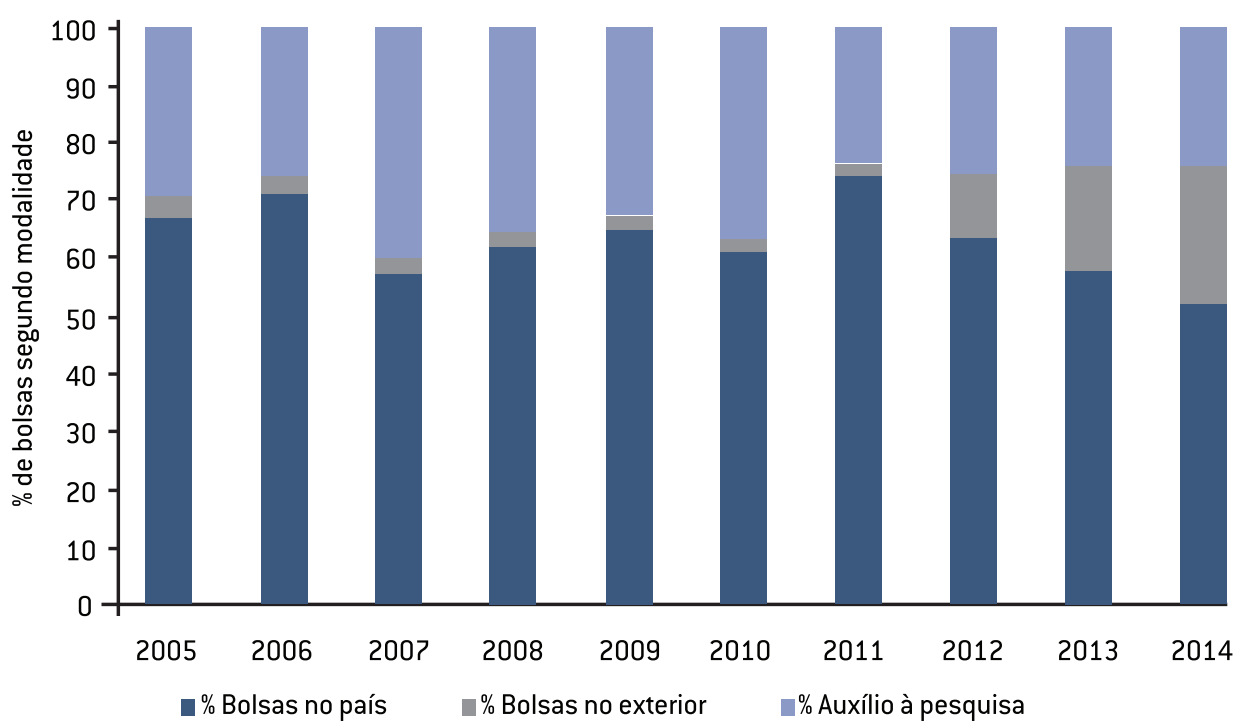

FIGURA 1 - Percentual de bolsas do CNPq segundo modalidade na área de Ciências da Saúde entre os anos de 2005-2014.

investimentos em bolsas de produtividade. Por outro lado, foram as subáreas com maior crescimento no período (276,0\% e $202,0 \%$, respectivamente). A Educação Física foi a sétima das subáreas em recebimento de bolsas de produtividade. Entretanto, foi a quarta em termos de crescimento (115,0\%; Figura 2). 


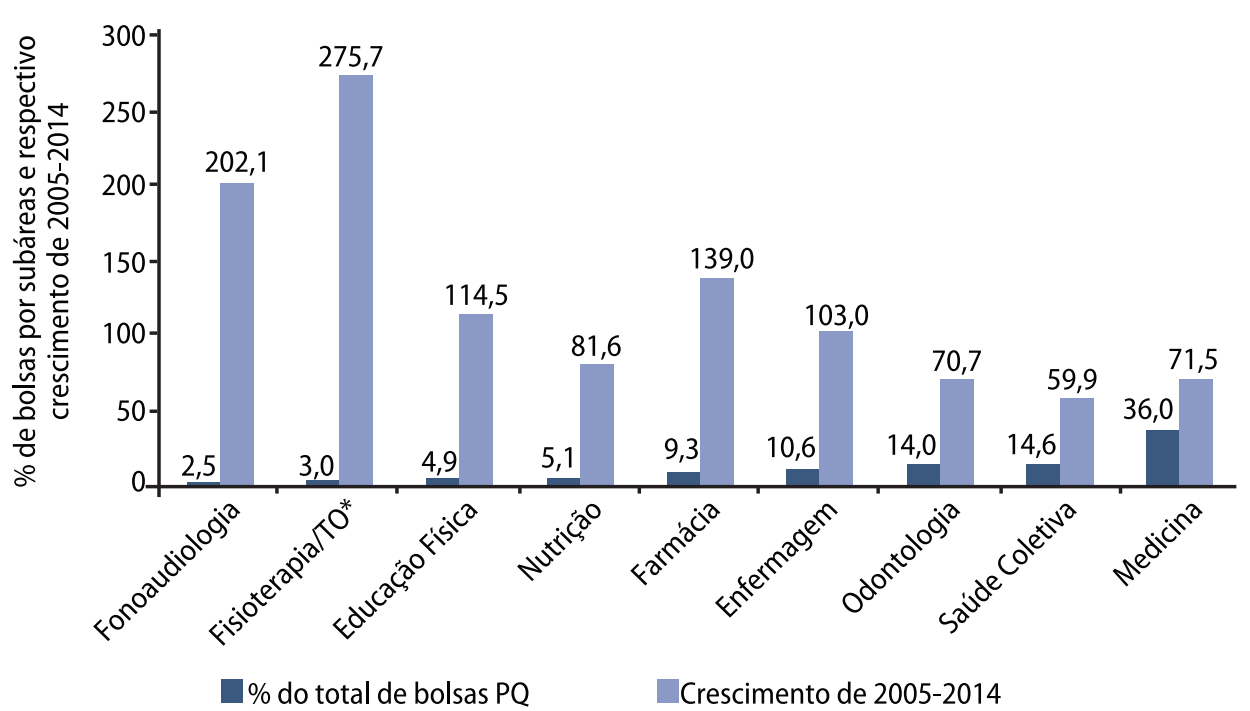

*TO: Terapia Ocupacional.

FIGURA 2 - Percentual de recebimento das bolsas entre as subáreas das Ciências da Saúde e o crescimento das verbas destinadas a elas entre 2005-2014.

Em relação aos valores brutos (em reais) investidos em todas as modalidades de bolsa, a subárea da Medicina foi a que mais recebeu verbas (R \$593.962.000,00), seguida pelas subáreas de Saúde Coletiva (R $\$ 302.474 .000,00)$ e Farmácia (R \$202.604.000,00). A Educação Física foi a sétima subárea em valores absolutos, tendo recebido ao longo do período avaliado $\mathrm{R} \$ 71.448 .000,00$. Do total dos investimentos nas áreas das Ciências da Saúde, a subárea que recebeu a maior proporção dos valores destinados aos financiamentos de bolsa ao longo dos anos foi a de Medicina (38,6\%), seguido da Saúde Coletiva (19,0\%) e da Farmácia com 12,7\% dos recursos. A Educação Física ocupou a sétima posição com 4,5\% (Figura 3).

Até o ano de 2014, haviam sido homologados 534 cursos de mestrado na área das Ciências da Saúde, sendo a maior proporção nas subáreas da Medicina (36,7\%), Odontologia (17,4\%), Saúde Coletiva (12,2\%), Enfermagem (11,0\%) e Farmácia $(9,9 \%)$. Na extremidade inferior, encontram-se os cursos de Educação Física (5,4\%), Nutrição (3,7\%), Fisioterapia/Terapia Ocupacional $(2,2 \%)$ e Fonoaudiologia (1,3\%). Quanto ao doutorado, haviam 328 cursos homologados em 2014, com maiores percentuais para os cursos de Medicina (49,1\%), Odontologia $(15,9 \%)$, Farmácia (9,5\%), Saúde Coletiva (8,5\%) e Enfermagem (7,9\%). Em seguida aparece a Educação Física, com 4,0\% dos cursos de doutorado, Nutrição (2,4\%), Fonoaudiologia (1,8\%) e Fisioterapia/Terapia Ocupacional (0,9\%).

Quanto ao percentual de crescimento de investimento nos cursos da área das Ciências da Saúde, verificou-se que houve aumento superior nas subáreas de Fisioterapia/Terapia Ocupacional (775,0\%) e Educação Física (738,0\%), seguidos por Farmácia (419,0\%) e Medicina (312,0\%). O menor crescimento observado foi na área da Saúde Coletiva (117,0\%; Figura 4).

Ao analisar os financiamentos de acordo com as regiões do país, verificou-se maior distribuição para a região Sudeste $(64,3 \%)$, seguida pelas regiões Sul $(15,4 \%)$ e Nordeste $(14,6 \%)$. Menores percentuais foram destinados para a região Centro -oeste $(4,1 \%)$ e especialmente para a região Norte $(1,6 \%)$. 


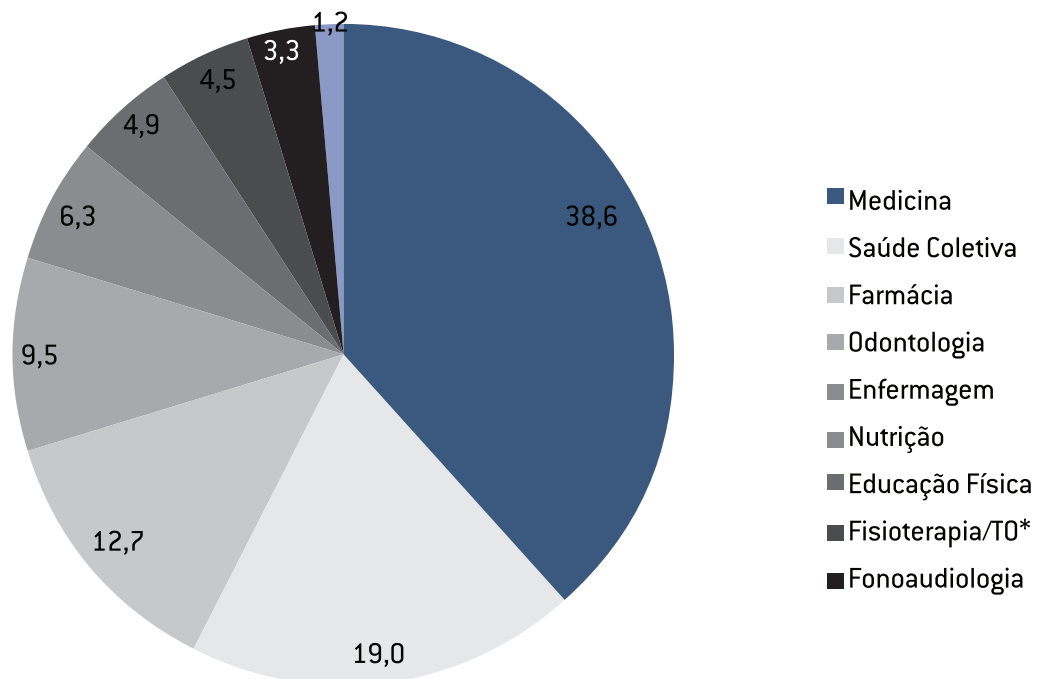

*TO: Terapia Ocupacional.

FIGURA 3 - Proporção de investimentos em bolsas de ensino e pesquisa do CNPq em cada uma das subáreas das Ciências da Saúde entre os anos de 2005-2014.

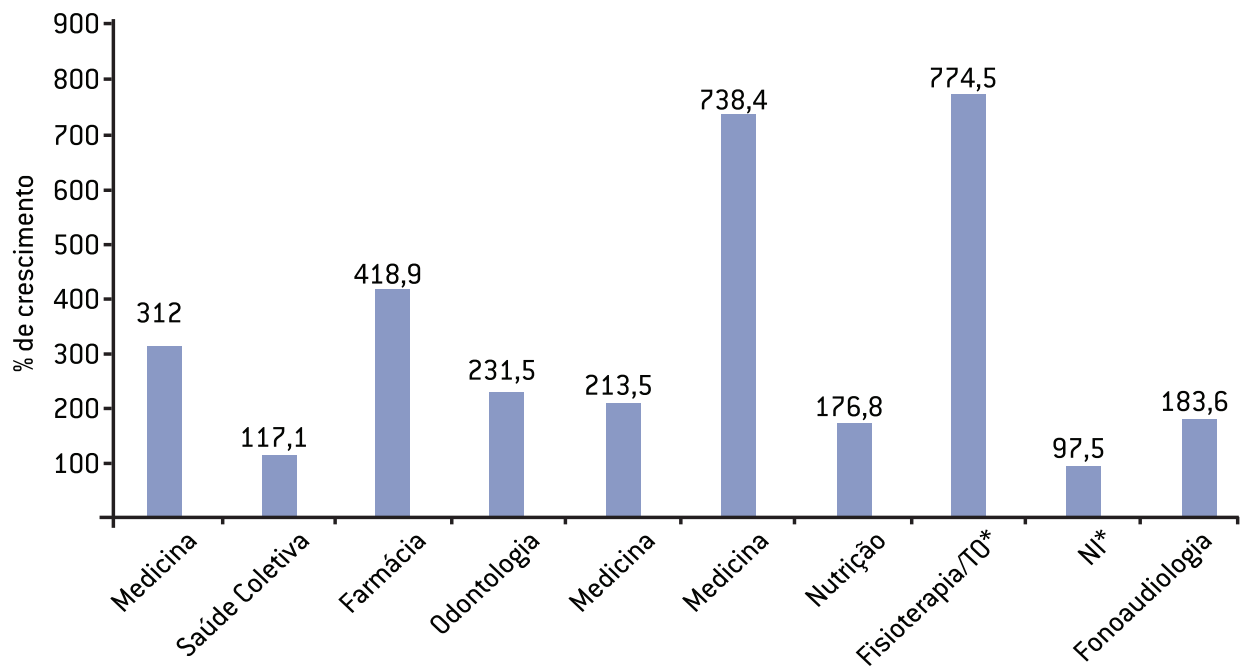

*TO: Terapia Ocupacional; * NI: Não informado.

FIGURA 4 - Percentual de crescimento em investimentos em cada subárea da área das Ciências da Saúde entre os anos de 2005-2014.

\section{Discussão}

O presente estudo apresenta um panorama dos financiamentos em pesquisa e concessão de bolsas de ensino e pesquisa pelo CNPq no período de 2005 a 2014. Além disso, destaca este investimento com relação à modalidade e ao tipo das bolsas, na grande área das Ciências da Saúde e, mais especificamente, na subárea da Educação Física. Ao longo dos últimos 10 anos, houve um aumento em valor absoluto nos investimentos através da concessão de bolsas no país, no exterior e auxílio à pesquisa, pelo CNPq.

Foi observada clara ascensão na concessão de bolsas para o exterior a partir do ano de 2012, com seu ápice no ano de 2014. Esse crescimento das bolsas internacionais se dá em função da implementação do programa "Ciência sem Fronteiras" do governo federal a partir de 2011, o qual tem como objetivo promover a consolidação, expansão e internacionalização da ciência e tecnologia, da inovação e da competitividade brasileira por meio do intercâmbio e da mobilidade internacional ${ }^{6}$. 
Notoriamente, há disponibilidade de uma quantidade relevante de bolsas, o que pode permitir uma prática contextualizada, bem como possibilitar ao professor o desenvolvimento da capacidade de replicar o processo de solucionar problemas 7 . Destacando que a concessão de bolsas não garante a qualidade do processo de formação acadêmica, sendo esta influenciada por outros fatores. Porém, a destinação de verbas pode auxiliar no incremento da construção do conhecimento, pois se observa que os indivíduos têm a oportunidade de aprender em cada experiência e isso ajuda a moldar a forma que venham a aprender, delineando sua trajetória acadêmica ${ }^{8}$. Ademais, o investimento em educação e empoderamento das universidades trazem consigo retornos para a sociedade através da qualificação de estudantes em questões políticas, técnico-científicas, jurídicas, culturais ${ }^{9}$, e, como na Educação Física, em aspectos relacionados à educação e promoção de saúde.

$\mathrm{O}$ investimento em bolsas de ensino e pesquisa do CNPq na Educação Física ainda é pequeno comparado as demais subáreas das Ciências da Saúde, embora tenha crescido substancialmente ao longo do período analisado neste estudo. A produção científica na subárea da Educação Física tem aumentado de forma significante, especialmente no período compreendido entre 2008-2012 ${ }^{10}$. Este fato pode estar relacionado a inúmeros aspectos, incluindo a quantidade de cursos de pósgraduação no Brasil reconhecidos pela CAPES. Tal diagnóstico reforça a necessidade de desenvolver maiores investimentos para a Educação Física, tendo em vista que esta área tem campo de atuação tanto na Saúde quanto na Educação. Embora se perceba esta demanda por maiores investimentos nesta subárea das Ciências da Saúde, sabe-se que outros aspectos, além de produção científica, devem ser considerados para destinação de verbas, tais como questões epidemiológicas, sociais, políticas e, a própria demanda requerida por cada subárea. Maiores investimentos podem possibilitar acesso a conhecimentos, formação profissional, desenvolvimentos de diversos projetos e programas de estudos que tendem a oportunizar melhores serviços à sociedade de um modo geral.

Neste contexto, num esforço para legitimar o ensino de uma Educação Física de qualidade, a UNESCO em conjunto com outras organizações nacionais e internacionais, criaram recentemente diretrizes que fornecem um marco de ação para apoiar gestores de políticas na reforma de políticas no campo de Educação Física, para acelerar o desenvolvimento de diversas dimensões do capital humano de uma forma original e abrangente ${ }^{11}$.

Ao analisar os valores de financiamento, percebe-se que a Medicina foi o curso que, dentro das áreas das Ciências da Saúde, recebeu mais investimentos (38,6\%) ao longo dos últimos 10 anos. Já a Educação Física foi a sétima área que mais recebeu investimentos (4,5\%). Em relação ao número de cursos de pós-graduação nas Ciências da Saúde, nota-se que a Medicina foi a subárea que mais tem cursos de pós-graduação; já a Educação Física foi a sexta subárea com maior número de cursos de pós-graduação. Desta forma, fica evidente que os investimentos em bolsas de ensino e pesquisa do CNPq tem sido proporcionais a quantidade de cursos de pós-graduação existentes em cada área, exceto nos cursos de Odontologia, que vem recebendo menos do que deveria, e a Saúde Coletiva, que recebe mais, considerando apenas a lógica de relação entre cursos de pós-graduação e investimento. Além disso, deve-se considerar que apenas uma fonte pública de financiamento foi acessada, podendo haver recebimento de verbas privadas pelas subáreas em questão.

Indica-se que desde a década de 70 vem ocorrendo um aumento no número de programas de pós-graduação brasileiros, em todas as áreas. Ao se analisar a produtividade da pós-graduação do país, verifica-se a publicação de cerca de 30,0\% em 
periódicos internacionais, enquanto a Educação Física o faz em apenas 5,0\% ${ }^{12}$ dos casos, indicando uma carência na área, que acaba por refletir nos demais tipos de publicações. Especificamente na Educação Física, alguns dos desafios permanecem, como a expansão do número de programas de pós-graduação sem perda de qualidade, aproximação com a graduação, preparação de profissionais pesquisadores, incremento da qualidade e quantidade de produção científica ${ }^{13}$.

Quando se analisa a média de crescimento dos investimentos no período de 2005-2014, a Fisioterapia/Terapia Ocupacional foi a subárea que mais cresceu em investimentos e a Educação Física a segunda. Apesar de a Medicina e a Saúde Coletiva serem as subáreas que mais receberam verbas ao longo dos últimos 10 anos, o crescimento proporcional em bolsas foi menor quando comparado com Educação Física e Fisioterapia/Terapia Ocupacional, provavelmente porque as duas primeiras subáreas historicamente já recebiam maiores financiamentos.

Quanto à relação do número de bolsas por região do país, o maior número de investimentos concedidos para a região Sudeste segue tendência de outra pesquisa, que descreve a proporção de projetos de pesquisas em vigência na área de Educação Física financiados pelo $\mathrm{CNPq}^{5}$. Os autores encontraram que a maior parte dos projetos está sendo realizados na região Sul e Sudeste (> que 60,0\%), e oposto a isto, a região Norte não possui nenhum projeto sendo desenvolvido na área. Sendo assim, parece claro que a concessão de bolsas seja maior nos locais com projetos de pesquisa vigentes. $\mathrm{O}$ investimento na formação de recursos humanos pode ser um meio para reduzir as desigualdades entre as regiões do país, já que há relação direta entre o número de doutores e programas de pós-graduação, consequentemente mais alunos, bolsas e recursos ${ }^{14}$. Ademais, indica-se que a parceria entre pesquisadores e universidades de diferentes locais do país pode ser uma estratégia interessante para diminuir as disparidades regionais.

Uma limitação do estudo está no fato que os dados foram extraídos somente da plataforma $\mathrm{CNPq}$, sendo que existem outras fontes de financiamento para a área das Ciências da Saúde. Além disso, em relação a busca dentro de cada subárea, salienta-se que alguns pesquisadores podem receber bolsas de outras áreas, bem como pesquisadores de outras áreas serem financiados na Educação Física. Ressalta-se que esta é uma análise inédita sobre o investimento na grande área das Ciências da Saúde e que pode contribuir para o conhecimento do direcionamento destas verbas ao longo dos anos.

Conclui-se que entre 2005-2014 as bolsas de produtividade em pesquisa e bolsas destinadas a realização de atividades no país foram as que mais receberam verbas do CNPq. Verificou-se também que a Educação Física foi a sétima subárea das Ciências da Saúde em termos de recebimento de verbas. Essas verbas foram maiores nas subáreas das Ciências da Saúde que apresentam maior número de cursos de pós-graduação. Embora o investimento na Educação Física ainda seja pequeno, esta subárea foi a segunda em termos de crescimento percentual de investimento ao longo do período, dado que pode estar relacionado ao aumento da produção científica na subárea.

Cabe salientar a necessidade de expansão das verbas destinadas às Ciências da Saúde como um todo, para que as subáreas possam seguir se desenvolvendo e expandindo seus conhecimentos. É também necessário dar destaque à disparidade no que tange a destinação de bolsas entre as regiões do país, tendo em vista o abismo existente entre a região Sudeste e Norte. 


\section{Contribuição dos autores}

LM Galliano teve a idéia original e participou de todas as etapas do estudo. LQ Corrêa, AEPS Sobrinho, DL Rosa, FMS Gomes e MP Melo realizaram coletas e análises de dados. MR Domingues e PC Hallal supervisionaram todas as fases do trabalho. Todos os autores participaram da escrita do artigo, dando contribuições intelectuais para o aprimoramento do mesmo. Todos os autores aprovaram a versão submetida para publicação.

\section{Referências}

1. Conselho Nacional de Desenvolvimento Científico e Tecnológico (CNPq). Áreas de conhecimento. <acesso em 24 de maio, 2015> http://www.cnpq.br/ documents/10157/186158/TabeladeAreasdoConhecimento.pdf

2. Kokubun E. Pós-Graduação em Educação Física. Rev Bras Educ Fís Esp. 2006;20:31-33.

3. Lazzarotti Filho A, Silva AM, Nascimento JV, Mascarenhas F. Modus Operandi da Produção Científica da EF: uma análise das revistas e suas veiculações. Rev Educ Fís/ UEM. 2012;23:1-14.

4. Coordenação de Aperfeiçoamento de Pessoal de Nível Superior (CAPES). Cursos recomendados. <acesso em 25 de mar, 2015>https://sucupira.capes.gov.br/sucupira/ public/consultas/coleta/programa/quantitativos/quantitativoAreaAvaliacao. jsf;jsessionid=LGzmWH42EWxm0SBhtmhLYDlY.sucupira-203

5. Silva ICM, Santin-Medeiros F, Bertapelli F, Coelho APS, Silva SG. Pesquisa em atividade física e saúde no Brasil: dimensão atual dos investimentos em projetos e bolsas de produtividade do CNPq. Rev Bras Ativ Fís Saúde. 2014;19(3):325-32.

6. BRASIL. Ministério da Educação. Programa Ciências sem Fronteiras. <acesso em 26 mar, 2015>http://www.cienciasemfronteiras.gov.br/web/csf/o-programa

7. Tani G. Professional preparation in physical education: Changing labor market and competence. Motriz. 2013;19:552-7.

8. Jarvis P. Learning to be a person in society. London: Routledge, 2009.

9. Fava-de-Moraes F. Universidade, inovação e impacto socioeconômico. São Paulo Perspec. 2000;14(3):8-11.

10. Andrade DC, López BA, Ramírez-Campillo R, Beltrán AR, Rodríguez RP. Bibliometric analysis of South American research in sports science from 1970 to 2012. Motriz. 2013;19:783-91.

11. Organização das Nações Unidas para a Educação, a Ciência e a Cultura (UNESCO). Diretrizes em educação física de qualidade (EFQ) para gestores de políticas. - Brasília: UNESCO, 2015.

12. Kokobun E. Pós-graduação em Educação Física no Brasil: indicadores objetivos dos desafios e das perspectivas. Rev Bras Cienc Esporte. 2003;24(2):9-26.

13. Tani G. Os desafios da pós-graduação em educação física. Rev Bras Cien Esporte. 2000;22(1):53-63.

14. Bortolozzi F, Gremski W. Pesquisa e pós-gradução brasileira - assimetrias. Rev Bras Pós Grad. 2004;1(2):35-52.

ENDEREÇO PARA

CORRESPONDÊNCIA

LEONY MORGANA GALLIANO

Imgalliano@gmaili.com
Luís de Camões, 625, Bairro Três Vendas

96055-630. Pelotas, RS

Telefone: (53) 3273-2752 